\title{
Universidad y diversidad funcional: aproximación a la inclusión en el ámbito universitario
}

\author{
Jessica Garabal Barbeira \\ Universidade de Coruña
}

\begin{abstract}
Resumen
Objetivos: Explorar las vivencias de los estudiantes universitarios con diversidad funcional y esbozar propuestas de mejora. Metodología: Estudio cualitativo realizado en una universidad gallega a estudiantes universitarios con diversidad funcional y un informante clave. Resultados: En las entrevistas individuales emergieron las categorías: "superación y aprendizaje", "frustración y cambio", "red social”, "recursos”, “entorno y barreras", "conocimientos" y "ocio"; y en el grupo de enfoque emergieron cinco nuevas categorías identificadas como propuestas de mejora. Conclusiones: Es necesario intervenir a favor de una comunidad universitaria inclusiva ya que los participantes se enfrentan de manera cotidiana con limitaciones que dificultan su correcto desarrollo.
\end{abstract}

Palabras clave: universidad, discapacidad, inclusión, diversidad funcional.

En la actualidad se presenta un descenso considerable del alumnado en el acceso a la universidad, hecho que perjudica a la igualdad en el contexto educativo. El ámbito universitario puede considerarse el lugar en el que las personas establecen lazos de unión, configuran su personalidad, participan socialmente, reflexionan sobre su identidad e intentan afianzar todos los aspectos de su persona según unos roles sociales aceptados. No se trata de una institución o estructura académica sin más, también supone un período y lugar de formación, de encuentro y de integración personal y social; en este marco sociotemporal y de desarrollo sistémico, es donde la persona es sujeto activo en su contorno (Luque Parra, Rodríguez Infante y Romero Pérez, 2005).

Los estudiantes pueden encontrarse a lo largo de su trayectoria académica algún tipo de barrera o dificultad tanto en el acceso a la documentación y al material audiovisual como en la relación docente-alumno; estas situaciones pueden llevar hacia el fracaso académico en un caso extremo, pero en otros muchos casos originan en el estudiante una situación de frustración al no poder llevar a cabo su labor universitaria de manera adecuada, normalizada y satisfactoria.

La población universitaria con discapacidad representa un grupo minoritario en este ámbito, prueba de esto es que sólo un 5,26\% de la población con discapacidad posee estudios superiores terminados (Instituto Nacional de Estadística, 2008). En la actualidad nos encontramos con que los estudiantes con diversidad funcional en las universidades de España representan un 13,16\% de la totalidad de personas con discapacidad (Instituto
Nacional de Estadística, 2008); por otro lado, la Comunidad Autónoma de Galicia cuenta con la totalidad de 413 estudiantes con diversidad funcional: 152 en la Universidad de A Coruña, 254 en la Universidad de Santiago de Compostela y 7 en la Universidad de Vigo (Fundación Universia, 2014). Las personas con diversidad funcional acceden en menor medida a los estudios universitarios y elijen principalmente los estudios a distancia a través de la Universidad Nacional de Educación a Distancia (UNED) (Peralta Morales, 2007), que contó con 8.876 alumnos con diversidad funcional matriculados durante el curso 2013-2014 (Universidad Nacional de Educación a Distancia, 2014).

Estos datos dejan entrever la existencia de dificultades en la realización de los estudios universitarios por parte de este colectivo, por este motivo se identifica la necesidad de llevar a cabo esta investigación. Se puede intuir la existencia de algún tipo de barrera u obstáculo que dificulta el desempeño satisfactorio de ocupaciones en las universidades presenciales (ocupaciones relacionadas con la educación y con la participación social), puesto que una gran cantidad de estudiantes decide realizar estudios en universidades no presenciales lo que indica que la relación con el ambiente puede ser un factor relevante en esta situación.

Por tanto, los objetivos que se han establecido para esta investigación son explorar las vivencias de los estudiantes con diversidad funcional en el ámbito universitario, determinar la percepción que poseen sobre la igualdad de oportunidades, conocer sus inquietudes y necesidades y, por último, establecer unas propuestas de mejora a favor de una comunidad universitaria inclusiva, en base a las necesidades detectadas por los propios participantes.

\section{Método}

\section{Participantes}

La muestra seleccionada para este estudio se encuentra compuesta por ocho estudiantes universitarios con diversidad funcional y un informante clave (técnico/a del Servicio de Atención a la Diversidad), todos ellos pertenecientes a una universidad del Sistema Universitario de Galicia (SUG). Los estudiantes que han participado poseen diferentes características relacionadas con la edad y las ramas educativas, como se puede observar en la siguiente tabla. 
Tabla 1.

Características de la muestra

Entrevistas individuales

\begin{tabular}{llll}
\hline & Sexo & Edad & Rama de conocimiento \\
\hline P1 & Mujer & 23 & Artes y humanidades \\
P2 & Mujer & 31 & Ciencias de la salud \\
P3 & Hombre & 21 & Ciencias sociales y juridicas \\
P4 & Hombre & 22 & Ingeniería y arquitectura \\
P5 & Hombre & 31 & Ingeniería y arquitectura \\
P6 & Mujer & 20 & Ingeniería y arquitectura \\
P7 & Mujer & 28 & Ingeniería y arquitectura \\
P8 & Hombre & 20 & Ciencias sociales y juridicas \\
\hline & & Grupo de enfoque \\
\hline & Sexo & Edad & Rama de conocimiento \\
\hline E1 & Mujer & 31 & Ciencias de la salud \\
E2 & Hombre & 22 & Ingeniería y arquitectura \\
E3 & Mujer & 20 & Ingeniería y arquitectura \\
E4 & Mujer & 28 & Ingeniería y arquitectura \\
\hline
\end{tabular}

\section{Instrumentos}

El estudio se basa en una metodología cualitativa con un diseño de investigación-acción participativa. El muestreo ha sido teórico y el método ha sido el descriptivo exploratorio con el objetivo de documentar el fenómeno del estudio.

Para la recogida de información se han utilizado técnicas observacionales y conversacionales; se ha llevado a cabo la triangulación de métodos de recogida de datos utilizando para ello entrevistas individuales con preguntas de naturaleza indagatoria y de tipología semiestructurada (a cada uno de los participantes y al informante clave) y un grupo de enfoque con el objetivo de exponer los resultados obtenidos en el análisis de datos, llevarlos a discusión y proponer medidas para resolver las necesidades detectadas.

\section{Procedimiento}

La información obtenida en las entrevistas ha sido transcrita, codificada de forma inductiva y agrupada en categorías, concentrando ideas, conceptos o temas similares (Rubin \& Rubin, 1995). A continuación se ha elaborado el diagnóstico del problema, presentado a los participantes en el grupo de enfoque, con el objetivo de agregar datos, validar información y confirmar hallazgos. Finalmente se han esbozado propuestas de mejora con el objetivo de crear el cambio; la implementación de éstas podría llevarse a cabo en posteriores investigaciones.

\section{Resultados}

\section{Resultados de las entrevistas individuales}

Superación y aprendizaje. Los participantes refieren que ambos sentimientos, superación y aprendizaje, confluyen, es decir, para los estudiantes implica tanto una superación estar cursando estudios universitarios como un aprendizaje, referido a la adquisición de conocimientos y de experiencias vitales, con la que se les proporciona un cimiento sobre el que encaminar su futuro, tanto profesional como personal: "Nada...aprender más que nada, lo que implica para cualquier persona, estudiar, desarrollarte profesionalmente, personalmente, sí, eso es lo que implica." P5.

Frustración y cambio. Participar en el ámbito universitario conlleva para ellos sentimientos de frustración debido al esfuerzo de enfrentarse con diversas barreras o necesidades que no se encuentran solventadas de antemano: "Para mí es un esfuerzo venir aquí todos los días y los profesores yo creo que no son conscientes de lo que realmente implica” P3. Narran también que la situación de cambio afecta relativamente a su desarrollo académico y personal, cambio al acceder a la universidad, conocer nuevos docentes, relacionarse con otras personas, entre otros: “...a decir verdad no crees que cuando vayas a llegar aquí, no te imaginas que va a ser tan duro" P7. Uno de los hechos que repercute en dicho cambio es la relación docente-alumno, desde el punto de vista de la mayoría de participantes, es necesaria una relación más cordial y menos institucionalizada: “...que tengan un poco de comunicación con el alumnado...un poco de ponerse en el lugar de la otra persona, un poco de empatía..." P6.

Red social: apoyo e impotencia. En base a la relación que mantienen con otros compañeros estudiantes manifiestan que, dependiendo del tipo de discapacidad se comportan de una manera determinada: "Probablemente la gente que no sabe lo que tienes, me trate diferente a mí que a alguna persona que se le ve el defecto físico" P7, prestando más atenciones a personas con discapacidades visibles, como la discapacidad física. En general, valoran la relación que mantienen con sus compañeros como "buena”: "Muy buena, bastante buena sí, la verdad es que cuando se presenta algún problema, son los primeros en tender la mano" P2. Sin embargo, hacen referencia a la actitud reticente que muestran algunos compañeros a la hora de realizar trabajos grupales con ellos, hecho que les provoca un sentimiento de impotencia: "Yo, lo que noto es que a la gente con discapacidad nos tratan diferente... por ejemplo los trabajos que hacemos, la gente es muy reacia a ir contigo, porque prefiere otra persona que no dé nada de dificultades para hacer un trabajo. $P 3$ ”.

Recursos. Por un lado, algunos participantes comentan cómo la universidad los ha apoyado, proporcionándoles los recursos necesarios: "Esta universidad sí. Creo que sí, hay cosas que es más complicado pero sí, yo creo que sí, por lo menos en todo lo que yo he querido participar la universidad siempre me ha apoyado" P5. Pero, por otro lado, hacen referencia a que durante su experiencia universitaria han vivido alguna circunstancia de falta de implicación por parte del profesorado en su proceso académico: “...sobre todo en el profesorado veo que no es la misma implicación. Por ejemplo, hay profesores que se implican más, otros tienen un interés menos acentuado que otros" $\mathrm{P} 2$.

Entorno y barreras. La mayoría de los participantes subrayan que se encuentran con barreras que repercuten en su correcto desarrollo académico: “En mi caso por ejemplo, si empiezo a nombrar todas las barreras arquitectónicas con las que me he encontrado pues, en mi caso no he tenido la misma igualdad que ha tenido otra gente entonces, en mi caso no, no es igualitario" $\mathrm{P} 4$, hecho que dificulta su participación en igualdad con el resto de compañeros. Por otro lado, refieren que es el entorno el que establece dichas barreras, ya que ser una persona con diversidad funcional no es una limitación, la 
limitación es que el entorno no se encuentra adaptado para desenvolver su labor académica de manera satisfactoria: "A veces su dependencia o $s u$ independencia pasa por los recursos que se pueden poner en marcha para ellos, entonces claro, ya no depende sólo de ellos, sino de todo lo que los rodea." Informante clave.

Conocimientos. Consideran que el hecho de relacionarse con sus compañeros y docentes de manera cotidiana les facilita la tarea de conocer la diversidad: "Bueno...la gente hasta que no tiene esto muy cerca no se va dando cuenta, pero bueno juegan con la ventaja de estar tú participando y también van conociendo tus necesidades y tus dificultades y vamos interactuando ambos, ellos van aprendiendo sobre todo y yo también aprendo." P5. Manifiestan discrepancias acerca de si existe o no desconocimiento sobre esta temática en ambos colectivos: "La mayoría de ellos no tienen ni idea, si no se lo explicas...cuando se lo explicas dicen. “'Ah!”, pero mientras tanto la mayoría de ellos no saben lo que implica." P7; “No sé qué decirte, con lo que vi de momento yo opino que sí, en mi caso lo supieron abordar." $P 8$.

Ocio universitario. Refieren que no participan en ningún tipo de actividad organizada por la comunidad universitaria, debido a diversos motivos: "Me parece que es una buena idea, aunque no participe. A lo mejor es cuestión de tener tiempo o querer hacer otras cosas simplemente, entonces no participo." P4; "No sé si en mi parte es falta de interés o que no tengo conocimientos por falta de información." E2.

\section{Resultados del grupo de enfoque}

Información previa sobre las necesidades de los estudiantes. Han indicado que la institución universitaria debería contar con información de los estudiantes matriculados previamente a que el curso académico comience: "Deberían avisar al profesorado antes de que empiece el curso, avisar con antelación para que se preparen, para que tengan una idea y poder facilitar un poco al alumno y al profesor" E1.

Propiciar el diseño universal. Sugieren y ven necesario propiciar el diseño universal, ya que es preciso eliminar las barreras arquitectónicas que suponen una dificultad para ellos: "Una universidad sin barreras, completamente sin barreras.” $P 1$.

Facilitar el acceso a la comunicación. Indican que es necesario facilitar el acceso a la comunicación a través de diferentes soportes o vías para poder acceder a documentación y conocimientos universitarios: "Le he pedido a los profesores que me facilitaran los apuntes de antemano y me decían que no me los podían facilitar, entonces tengo que molestar a mis compañeros, que son personales y a la hora de leerlo cada uno lo entiende a su manera." E1.

Formación en temas relacionados con la discapacidad. Se evidencia la necesidad de potenciar la oferta de formación en temas relacionados con la discapacidad, con la que se adquieran actitudes y aptitudes sobre cómo tratar o acometer diferentes situaciones: "Yo pienso que eso va en la personalidad de cada uno, el caso es tener interés, cuando aparece el problema que te intenten ayudar de la mejor manera." E2.

Ocio universitario para todos. Sugieren la introducción gradual de adaptaciones y más información para fomentar la inclusión de personas con diversidad funcional en actividades de ocio enmarcadas en el ámbito universitario: "Creo que hay cosas que se podrían cubrir por ejemplo, si yo quiero hacer atletismo o el equivalente a atletismo que hay para minusválidos, te ponen una silla de correr o algo así, que tengan una silla que a lo mejor te cueste un pastizal y que a lo mejor nunca se va a usar pues no; el fútbol por ejemplo creo que para los ciegos les ponen una campanilla o algo así en la pelota, pues que tengan una pelota de esas que a lo mejor no es un gran desembolso de dinero, creo que no les costaría nada tenerla ahí, por si se diera el caso." E3.

\section{Discusión}

El presente estudio sugiere que es necesario intervenir a favor de una comunidad universitaria inclusiva. Después de analizar los datos se observa que los estudiantes universitarios con diversidad funcional que participaron en esta investigación se encuentran diariamente con limitaciones o barreras que repercuten en su correcto desarrollo en el ámbito académico. Explorando las vivencias de los estudiantes con diversidad funcional en el ámbito universitario se observa cómo el hecho de estar cursando estudios superiores supone una superación personal y profesional a la vez que implica una adquisición de conocimientos enriquecedora para su futuro, Moriña Díez y Molina Romo (2011) consideran que los estudios universitarios contribuyen en sí mismos al crecimiento personal e intelectual, lo que en algunos casos se traduce en una mejora en el grado de autonomía.

Los compañeros actúan como soporte facilitando en la medida de lo posible la participación en todas las ocupaciones pertenecientes y características de este ámbito. Novo-Corti et al. (2011) indican que los estudiantes universitarios muestran una actitud positiva hacia sus compañeros con diversidad funcional y un mayor grado de sensibilización hacia la discapacidad. En la tesis doctoral de Martínez Martín (2010) se refleja que los estudiantes muestran una actitud generalmente positiva hacia la diversidad funcional.

La institución universitaria funciona como un pilar muy importante en cuanto a la suministración de recursos y resolución de problemáticas aunque se identifican ciertas carencias en relación con la falta de conocimientos sobre temas relacionados con la discapacidad, hecho que disminuye las estrategias para la detección de necesidades de los estudiantes y por consiguiente, la resolución de problemáticas. Debido a la antedicha situación Moriña Díez, López Gavira, Melero Aguilar, Cortés Vega y Molina Romo (2013) y señalando como principal barrera la falta de formación por parte del profesorado, identifican la necesidad de dotar de una mayor información y formación al personal de la institución para atender la diversidad. Identificaron esa misma necesidad Castellana Rosell y Sala Bars (2005), 
Hadjikakou y Hartas (2008), Moswela y Mukhopadhyay (2011) y Sánchez Palomino (2009) que subrayan que el profesorado debería formarse para proporcionar una respuesta de calidad a esto.

En la presente investigación se valora de manera muy positiva la convivencia tanto con docentes como con alumnos; esta convivencia, según Higbee, Siaka, y Bruch (2007) es reconocida por los estudiantes como una experiencia educativa enriquecedora, puesto que es favorable a la hora de facilitar la adquisición de conocimientos sobre la manera de enfrentarse con ciertas situaciones. Martínez Martín (2010) indica que la experiencia académica en situación de integración educativa afecta favorablemente en la actitud de los estudiantes hacia las personas con diversidad funcional.

El entorno se identifica como la fuente que aporta parte de las barreras con las que se encuentran de manera cotidiana, Fernie y Henning (2006) y Oliver y Barnes (2010) explicaron que las barreras que se encuentra el alumnado están en el ambiente y no en el individuo. Por tanto se considera que si esas barreras no existiesen, la diversidad funcional no debería repercutir en el desarrollo autónomo de las personas en el ámbito universitario. Moriña Díez y Molina Romo (2011) concluyen que las barreras arquitectónicas que se encuentran en los centros, incluyendo las aulas que en muchos casos están obsoletas, carecen de posibilidad de adaptación para los alumnos con discapacidad o no cuentan con una acústica adecuada; a la vez que Novo-Corti et al. (2011) opinan que la universidad no cuenta con los recursos precisos para impartir docencia a alumnos con discapacidad y que en sus edificios existen muchas barreras arquitectónicas.

Se ha detectado la necesidad de propiciar el diseño universal con el objetivo de alcanzar la igualdad de oportunidades en el acceso al ámbito universitario. En la actualidad se están adaptando cada una de las instalaciones que suponen algún tipo de barrera o restricción para el individuo; pero cuenta con verdadera importancia la necesidad de diseñar todas las instituciones basándose en los principios de accesibilidad universal, desde esta perspectiva no se adaptan las barreras a las necesidades individuales, sino que desde la creación de la institución se piensa en las necesidades del colectivo favoreciendo con este principio de diseño universal el desempeño ocupacional no sólo de los estudiantes con diversidad funcional, sino de toda la sociedad.

Aunque la mayor parte de los participantes expresaron su deseo de ser tratados como un alumno más también reconocen que tienen algunas necesidades a las que hay que dar respuesta. Posee relevancia que deben tener un acceso completo e igualitario a la información y a la documentación, Moriña Díez et al. (2013) subrayan en su estudio que en numerosas ocasiones los docentes no se mostraron predispuestos a facilitar el antedicho acceso. El desconocimiento sobre temas relacionados con la diversidad puede ser un factor a tener en cuenta para que sucedan estas situaciones en el ámbito universitario.

La existencia de una relación más distante con los docentes que en los estudios previos puede perjudicar a la adecuación de los estudiantes al ámbito académico en el que se encuentran inmersos y repercutir en la adaptación a este medio, haciendo que se produzca con mayor lentitud y dificultad. Tinklin, Riddel, y Wilson (2004) mencionan que la universidad en ocasiones, no está lo suficientemente preparada para incluir al alumno con diversidad funcional; a pesar de que existen normativas que regulen el derecho de este alumnado para recibir una educación de calidad y en igualdad de oportunidades, con las adaptaciones curriculares que sean necesarias. Moriña Díez et al. (2013) identificaron más barreras que ayudas en la experiencia académica de sus participantes.

Después de explorar las vivencias de los estudiantes universitarios con diversidad funcional tratando de aproximarse a su opinión sobre el desarrollo en el ámbito universitario, se han podido conocer y esbozar algunas de sus inquietudes y necesidades, entre las que destacan la necesidad de fomentar la formación en temas relacionados con la diversidad funcional, propiciar el diseño universal, aportar información previa de las necesidades de los estudiantes, facilitar el acceso a la comunicación y promover la existencia de un ocio para todos con la finalidad de alcanzar una comunidad universitaria inclusiva.

Una de las premisas de la Terapia Ocupacional, profesión sociosanitaria, es que el hombre es un ser activo que a través de la historia, estuvo y está haciendo continuamente. En el ámbito social los terapeutas ocupacionales dirigen su actividad profesional a potenciar el desempeño ocupacional del individuo para que este alcance la máxima participación social.

Por tanto, las intervenciones de los terapeutas ocupacionales en el ámbito de esta investigación están dirigidas a favorecer la relación entre el individuo, el entorno y la ocupación con el fin de promover un desarrollo autónomo para los estudiantes en un ambiente que favorezca la participación, es decir, en un ambiente inclusivo.

\section{Conclusiones}

Los resultados obtenidos en esta investigación ponen de manifiesto la necesidad de intervenir a favor de una comunidad universitaria inclusiva debido a la existencia de barreras que dificultan, o incluso imposibilitan, un desempeño ocupacional adecuado y satisfactorio en el ámbito universitario.

Tener acceso a una educación de calidad y formarse en una titulación que uno elija es un proceso que cuenta con gran importancia y repercusión para el desarrollo personal futuro. Participar libremente en la educación es un derecho para la ciudadanía, no sólo para una parte de los ciudadanos, por este motivo es relevante garantizar esa participación inclusiva $y$ en igualdad de oportunidades en el ámbito universitario respetando uno de los derechos fundamentales como es el de la educación.

\section{Referencias}

Bausela Herreras, E. (2002). Atención a la diversidad en la educación superior. Profesorado, revista de currículum y formación del profesorado, 6 , 1-9. 
Castellana Rosell, M., y Sala Bars, I. (2005). La universidad ante la diversidad en el aula. Aula $\operatorname{abierta(85),57-84.~}$

Diario Oficial de Galicia. (21 de Diciembre de 2011). DECRETO 229/2011. Obtenido el 8 de Marzo de 2014, de http://www.xunta.es/dog/Publicados/2011/20111221/ AnuncioC3F1-151211-9847_es.pdf

Fernie, T., \& Henning, M. (2006). From a disabling world to a new vision. Towards inclusive learning in higher education: Developing curriculum for disabled students. Essex, 23-32.

Fundación Universia. (19 de Febrero de 2014). Guía de atención a la discapacidad en la universidad 2014. Obtenido el 8 de Marzo de 2014, de Fundación Universia: http://www.fundacionuniversia.net/fichero?i $\mathrm{d}=2315$

Gómez Lillo, S. (2003). La ocupación y su significado como factor influyente de la identidad personal. Revista Chilena de Terapia Ocupacional(3), 43-47.

Hadjikakou, K., \& Hartas, D. (2008). Higher education provision for studients with disabilities in Cyprus. High Educ, 103-119.

Higbee, J. L., Siaka, K., \& Bruch, P. L. (2007). Assesing our commitment to multiculturalism: Student perspectives. Journal of College Reading \& Learning, 37(2), 7-25.

Instituto Nacional de Estadística. (2008). Porcentaje de personas con discapacidad de 16 y más años que están realizando algún estudio según tipo de estudio por sexo. Recuperado el 26 de Mayo de 2014, de Instituto Nacional

Estadística: http://www.ine.es/jaxi/tabla.do?path=/t15/p 418/a2008/hogares/p01/modulo3/10/\&file=02054a.px\&t ype $=$ pcaxis $\& \mathrm{~L}=0$

Kielhofner, G. (1995). El modelo de ocupación humana. Revista informativa de la Asociación Profesional Española de Terapeutas Ocupacionales(12), 3-9.

Luque Parra, D. J., Rodríguez Infante, G., \& Romero Pérez, J. F. (2005). Accesibilidad y Universidad. Un estudio descriptivo. Intervención Psicosocial, 14(2), 209-222.

Martínez Martín, M. Á. (2010). Los estudiantes con discapacidad en la universidad de Burgos. Recuperado el 2 de Mayo de 2014, de Universidad de Burgos: http://hdl.handle.net/10259/140
Moriña Díez, A., \& Molina Romo, V. (2011). La universidad a análisis: Las voces del alumnado con discapacidad. Revista de enseñanza universitaria(37), 23-35.

Moriña Díez, A., López Gavira, R., Melero Aguilar, N., Cortés Vega, M., \& Molina Romo, V. (2013). El profesorado en la universidad ante el alumnado con discapacidad ¿Tendiendo puentes o levantando muros? Revista de docencia universitaria, 11(3), 423-442.

Moswela, E., \& Mukhopadhyay, S. (2011). Asking for too much? The voices of students with disabilities in Botswana. Disability \& Society, 26(3), 307-319.

Novo-Corti, I., Muñoz-Cantero, J., \& Calvo-Porral, C. (2011). Análisis de las actitudes de los jóvenes universitarios hacia la discapacidad: un enfoque desde la teoría dela acción razonada. RELIEVE, 17(2), 1-26.

Oliver, M., \& Barnes, C. (2010). Disability studies, disabled people and the struggle for inclusion. British Journal of Sociology of Education, 31(5), 547-560.

Peralta Morales, A. (Octubre de 2007). Libro blanco sobre universidad y discapacidad. Recuperado el 8 de Marzo de 2014, de Real Patronato sobre Discapacidad: http://sid.usal.es/idocs/F8/FDO20244/Lib roBlancosobreuniversidadydiscapacidad2.pdf

Rubin, H., \& Rubin, I. (1995). Qualitative interviewing: The art of hearing data. Thousand Oaks: Sage.

Sánchez Palomino, A. (2009). Integración educativa y social de los estudiantes con discapacidad en la Universidad de Almería. (Vol. 128). Universidad de Almería.

Simó Algado, S. (2006). El Modelo Canadiense del Proceso del Desempeño Ocupacional. TOG (A Coruña)(3), 1-18.

Tinklin, T., Riddel, S., \& Wilson, A. (2004). Policy and provison for disabled students in higher education in Scotland and England: The current state of play. Studies in Higher Education, 29(5), 637-657.

Universidad Nacional de Educación a Distancia. (26 de Marzo de 2014). Información estadística del centro de atención a universitarios con discapacidad. Madrid, España.

World Federation of Occupational Therapists. (2010). Statement on Occupational Therapy. Retrieved from World Federation of Occupational Therapists. 